Chondromyxoid fibroma of skull base: A tumour prone to local recurrence

T W H Shek; W C G Peh; G Leung

The Journal of Laryngology and Otology; Apr 1999; 113, 4; ProQuest Medical Library

pg. 380

\title{
Chondromyxoid fibroma of skull base: a tumour prone to local recurrence
}

\author{
T. W. H. SHek, M.B., B.S., F.R.C.P.A.*, W. C. G. PeH, M.B., B.S., F.R.C.R.†, G. LEUNG, M.B., B.S. F.R.C.S.(Ed.)
}

\begin{abstract}
Chondromyxoid fibroma of the skull base is extremely uncommon. Sometimes involvement of the nasal cavity may occur and the patients may present with nasal symptoms. The biological behaviour of this tumour has not been well studied, primarily because of the limited number of reported cases and the short duration of followup. We report a histologically confirmed case of chondromyxoid fibroma of the skull base that recurred repeatedly over a 10-year period after the initial operation. Histologically it showed identical morphology to the original tumour with no evidence of histological progression or dedifferentiation. Ultrastructurally, the spindle tumour cells in the fibromyxoid area showed dual chondroblastic and fibroblastic differentiation, suggesting that these spindle fibroblastic cells and the better differentiated chondroid cells were of the same cell type with different histological morphology.
\end{abstract}

Key words: Chondromyxoid fibroma; Skull base; Nasal cavity; Electron microscopy

\section{Introduction}

Chondromyxoid fibroma (CMF) is one of the rarest benign bone tumours. It typically affects young adults with a predilection to involve the metaphysis of long bones of the lower extremities. Although thorough curettage may be able to cure a certain percentage of chondromyxoid fibromas, there is a relatively high incidence of recurrence after this form of treatment, which varies from 12.5 to 25 per cent (Zillmer and Dorfman, 1989; Unni, 1996). By virtue of the anatomical location. CMF of the skull base can be technically very challenging to the surgeon. Not uncommonly the tumour cannot be completely removed, and is therefore prone to local recurrence. Review of the literature shows that there have been fewer than 20 reported cases of CMF of the skull base (Table I), many of which do not have follow-up data. There have been only two documented cases of tumour recurrence (Frank et al., 1987; Keel et al., 1997). Recently we encountered a case of CMF of the skull base, which recurred repeatedly after the initial operation. The clinical, radiological, histological and ultrastructural findings of the case form the basis of this report.

\section{Case report}

The patient was a Chinese girl who presented at the age of six with a two-year history of frequent epistaxis and difficulty in breathing at night. Physical examination

TABLE I

SUMMARY OF REPORTED CASES OF CHONDROMYXOID FIBROMA OF THE SKULL BASE

\begin{tabular}{|c|c|c|c|c|c|}
\hline Authors & Location & Sex & Age & Treatment & Outcome \\
\hline Morikawa et al. (1987) & Ethmoid & $\mathrm{F}$ & 8 & Surgery & NED, 6 months \\
\hline Maruyama et al. (1994) & Occipital bone & NA & NA & Surgery & NA \\
\hline Jaffe and Lichenstein (1948) & Temporal bone & M & 67 & Surgery & NA \\
\hline Kitamura et al. (1982) & Occipital bone & $\mathrm{F}$ & 47 & Surgery & NA \\
\hline Dahlin (1956) & Occipital bone & $\mathrm{F}$ & 47 & Surgery & NED, 8 weeks \\
\hline Inoue et al. (1978) & Mastoid & $\mathrm{M}$ & 48 & Surgery & NA \\
\hline Keel et al. (1997) & Sphenoid-occipital bone & $\mathrm{F}$ & 68 & $\begin{array}{l}\text { Curettage twice, } \\
6100 \text { rads }\end{array}$ & $\begin{array}{l}\text { Recurred at } 6 \text { months, } \\
\text { NED at } 20 \text { months }\end{array}$ \\
\hline Patino-Cordoba et al. (1998) & Clivus & F & 41 & Curettage twice & Recurred at 1 year \\
\hline Patino-Cordoba et al. (1998) & Mastoid & M & 20 & Curettage & NA \\
\hline Wu et al. (1998) & Sphenoid $\times 3$ & NA & NA & NA & NA \\
\hline Wu et al. (1998) & Ethmoid $\times 2$ & NA & NA & NA & NA \\
\hline Shek et al. (present study) & Sella/Nasal cavity & $\mathrm{F}$ & 16 & $\begin{array}{l}\text { Curettage four times, } \\
5000 \text { rads }\end{array}$ & $\begin{array}{l}\text { Multiple recurrences } \\
\text { over } 10 \text { years }\end{array}$ \\
\hline
\end{tabular}

NED: No evidence of disease; NA: Not available.

From the Department of Pathology*, Diagnostic Radiology† and Surgerył, University of Hong Kong, Queen Mary Hospital, Hong Kong. Accepted for publication: 21 December 1998. 
showed a large soft tissue mass occupying both nasal cavities. There was telecanthus and her visual acuity was impaired bilaterally (right $6 / 24$ and left $6 / 18$ ). There was no cranial nerve deficit and systemic examination was unremarkable. Unfortunately, the initial presenting images were not available for review.

Craniofacial excision of the tumour was performed, and a $4 \mathrm{~cm}$ whitish tumour mass was excised. She remained asymptomatic until the age of 10 when she presented with deteriorating visual acuity. Physical examination showed bilateral optic nerve atrophy. Radiography showed sclerosis of the base of the skull, involving the sphenoid body, superior nasal cavity and posterior ethmoid sinuses. Computed tomography (CT) confirmed the presence of a suprasellar lobulated mass arising from the sphenoid and ethmoid sinuses. The mass was heavily calcified and displayed marked contrast enhancement.

Subsequently she had three more operations for recurrent disease. Multiple CT scans were also performed for evaluation, documenting each episodes of tumour recurrence. CT appearances were always similar, consisting of a lobulated mass with extension to the temporal fossa, posterior orbits and posterior nasopharynx (Figure 1a and b). Apart from the intracranial component, there was also extensive invasion into the left maxillary, right frontal and right anterior ethmoidal sinuses. The first reoperation was performed four years after the initial resection, using a combined transcranial transphenoidal approach. Total resection of the tumour was impossible due to the proximity of the posterior circulation complex, and subtotal resection was performed. Debulking of the recurrent tumour via the previous bifrontal craniotomy was again performed seven years after the initial operation. She then developed bilateral blindness due to optic nerve compression as well as symptoms or raised intracranial pressure. In view of the significant surgical risk and the reluctance of the patient for further surgery, she was given a course of external irradiation with a total dose of 5000 rads, but there was no response. The tumour persisted and she finally agreed to undergo the fourth

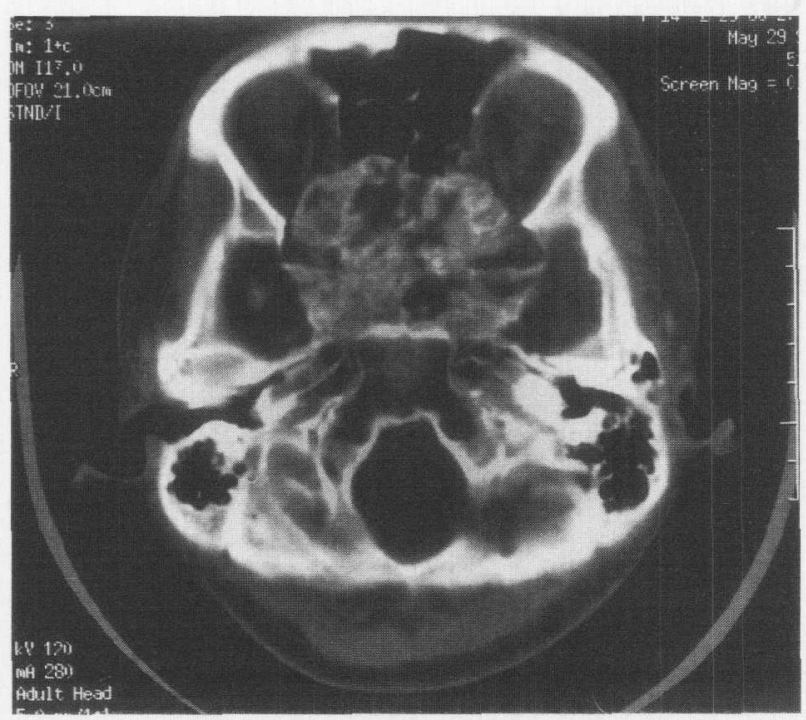

FIG. 1a

Axial CT scan taken at the level of the petrous temporal bone shows a heavily-mineralized tumour arising from the ethmoid and sphenoid sinuses. It is well-defined with lobulated contours, and is traversed by numerous ridges. It extends laterally into both temporal fossae and anterolaterally into the posterior orbital cavities.

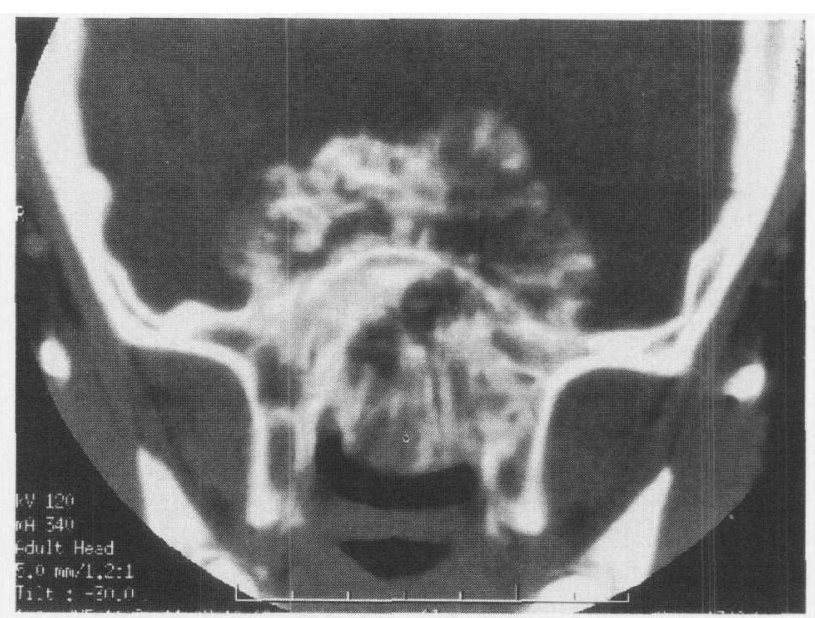

FIG. 1b

Direct coronal CT scan taken through the sphenoid sinus shows the suprasellar extension of the lobulated mineralized tumour. The pituitary fossa and surrounding structures are completely obliterated by the tumour. Inferior extension into the posterior nasopharynx is noted.

and the latest operation which was performed at the age of 16, 10 years after the initial presentation. Because the tumour was closely adherent to the thalamus and brain stem, only subtotal resection was attempted.

Histological examination of the resected tumour tissue at various operations over the years showed remarkably similar histology with no evidence of histological progression or dedifferentiation. The tumour showed a prominent lobular pattern of growth. These lobules were of irregular sizes, and they were bordered by fibrous connective tissue septa. The lobules showed a hypocellular centre with a rim of a more hypercellular zone at the periphery (Figure 2). The hypocellular centre had a loose fibromyxoid stroma in which scattered isolated multipolar stellate cells were found. In areas, these lobules contained better differentiated chondroid areas where cells with clear lacunar spaces were found (Figure 3).

Immunohistochemical studies showed that the vast majority of the tumour cells, including the spindle fibroblastic cells, were strongly positive for $\$ 100$ protein. They were negative for a variety of cytokeratins (CAM 5.2, AE1/3 and MAK6) as well as EMA.

The piece of tissue for electron microscopical studies mainly consisted of fibromyxoid areas. Ultrastructural examination showed scattered isolated spindle or stellate cells in a background of loosely-arranged collagen fibres. The spindle or stellate tumour cells had small cytoplasmic projections on their surfaces, and there were abundant glycogen particles in their cytoplasm (Figure 4), features in keeping with chondroid differentiation. In addition. they also possessed prominent rough endoplasmic reticulum in their cytoplasm as well as occasional small dense bodies comprising aggregates of microfilaments, features suggestive of fibroblastic/myofibroblastic differentiation (Figure 5).

\section{Discussion}

When occurring in the metaphysis of long bone in a young adult, chondromyxoid fibroma should not pose much of a diagnostic or therapeutic problem to the surgical pathologist, radiologist or surgeon. The major diagnostic challenge to the histopathologist is not to over-diagnose this tumour as chondrosarcoma, a point which has been 


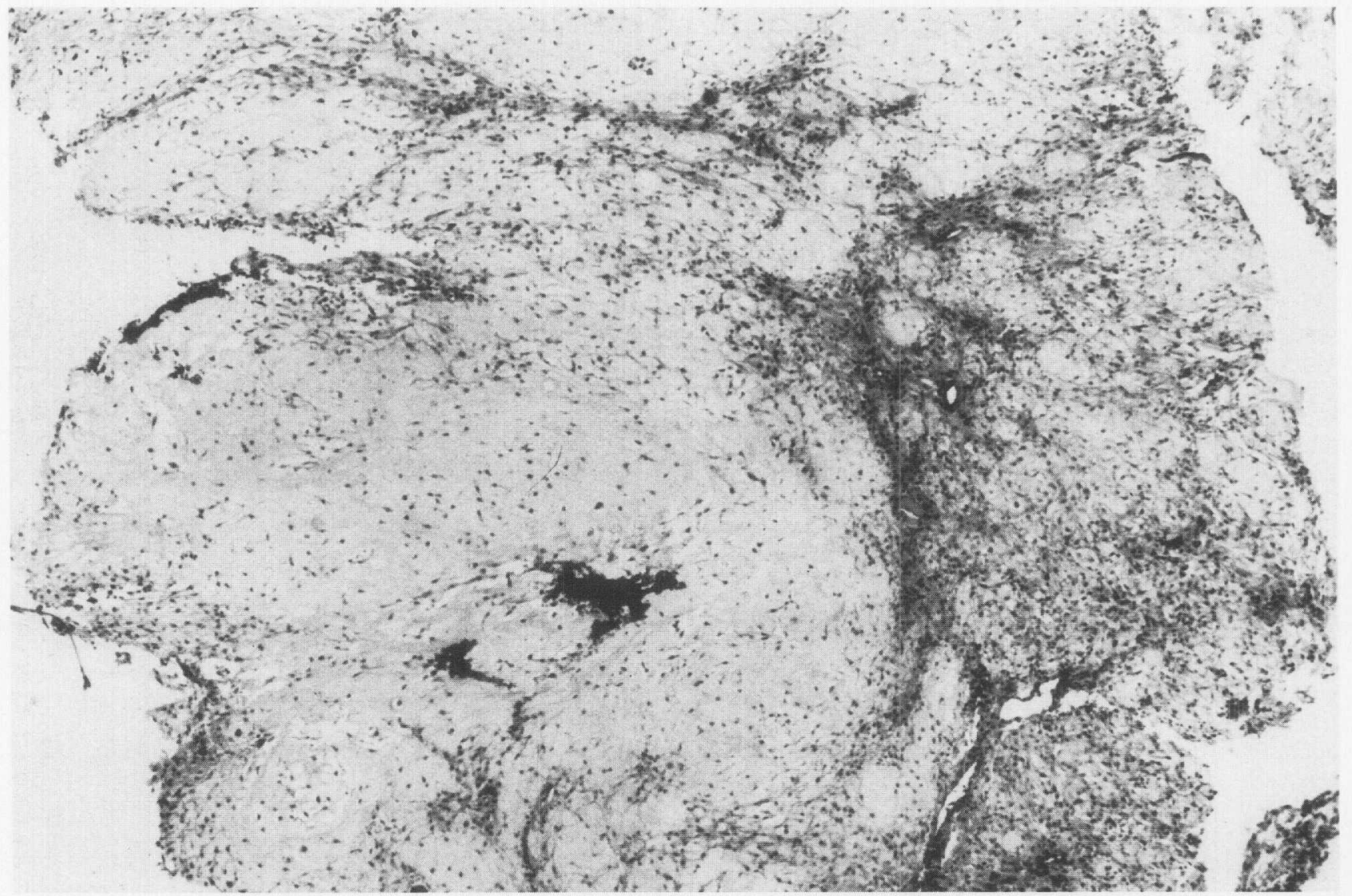

FIG. 2

The tumour shows a lobulated architecture with a peripheral rim of hypercellularity. $(\mathrm{H} \& \mathrm{E} ; \times 50)$

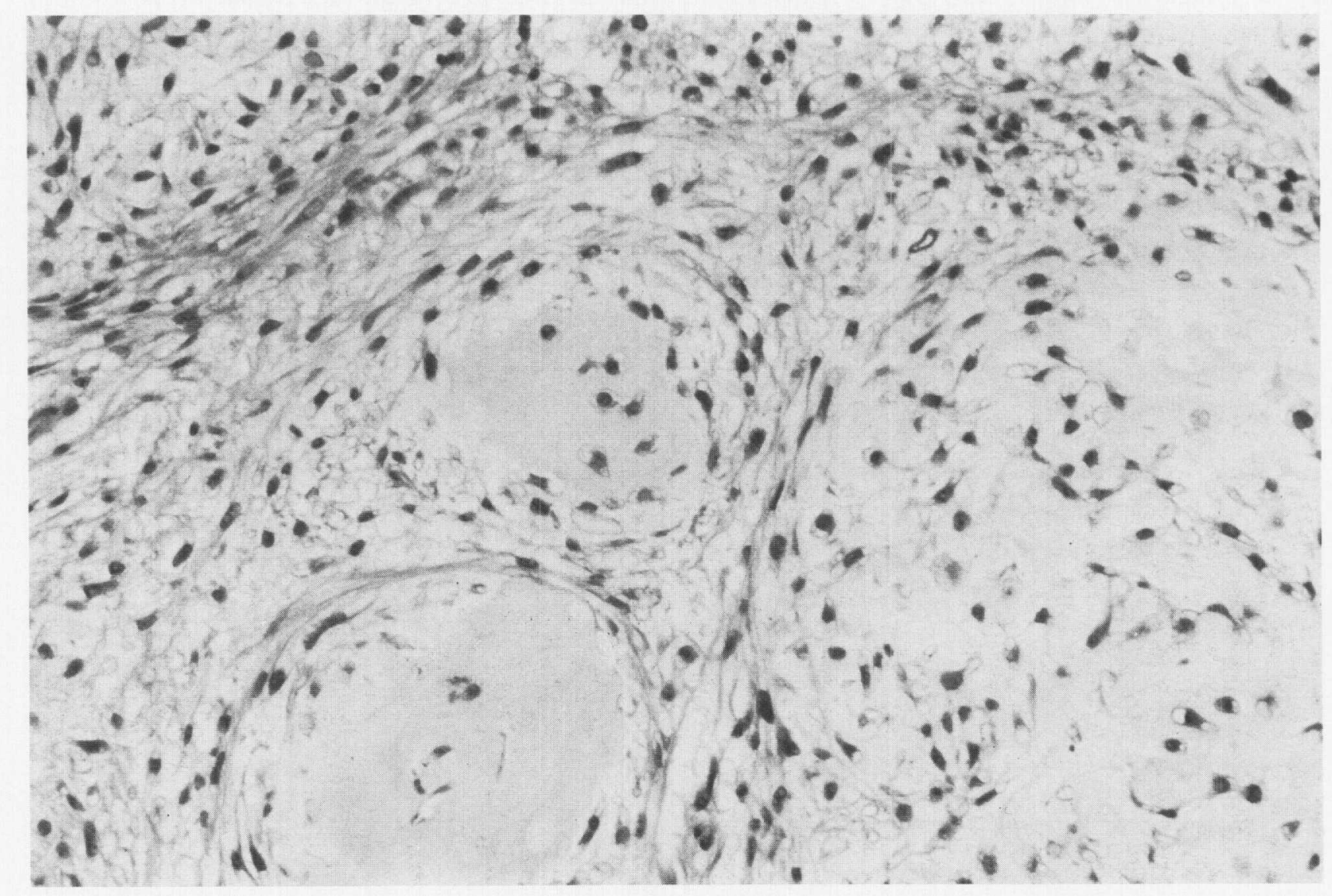

Better differentiated chondroid areas with adjacent spindle cells. (H \& E; × 250) 


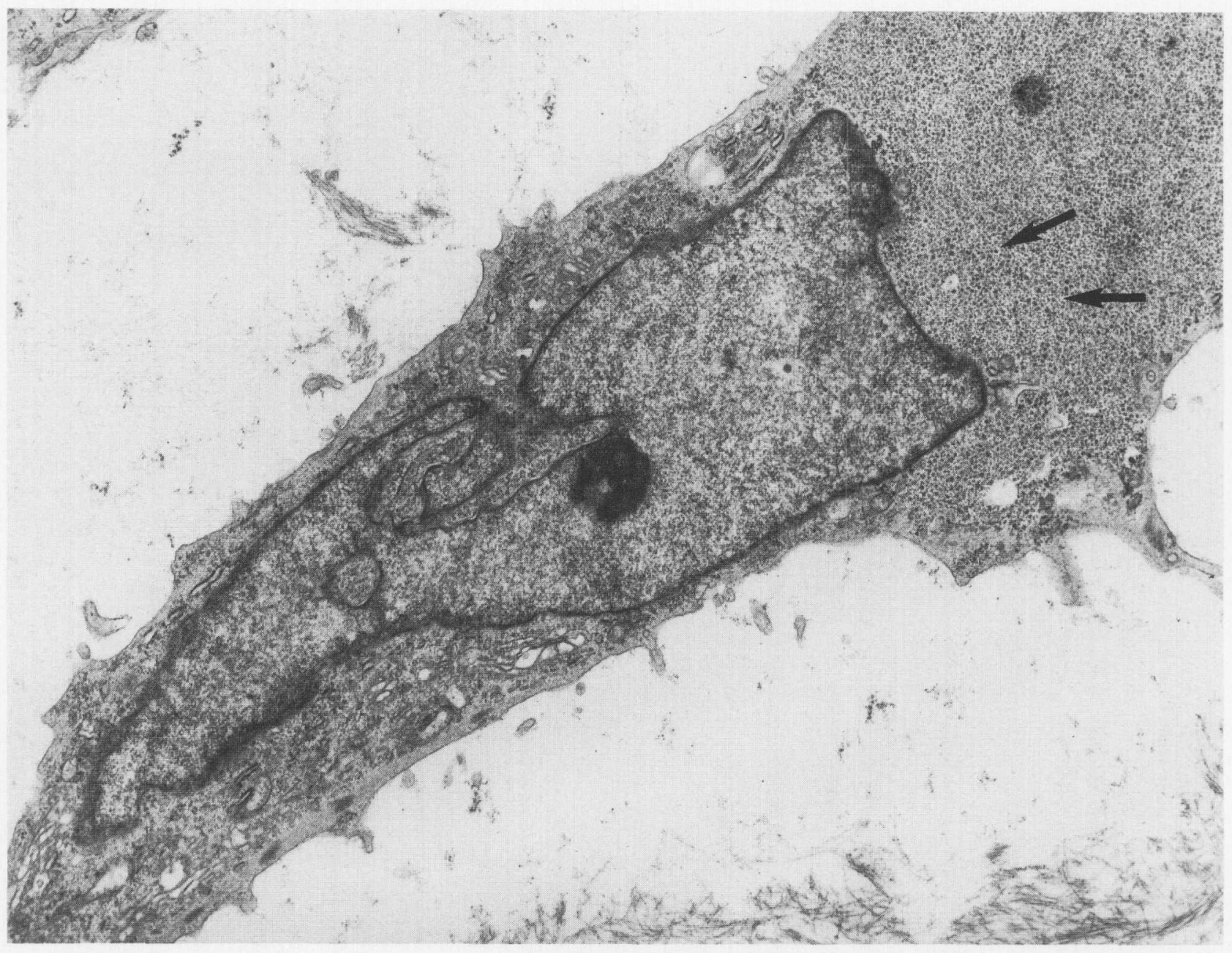

FIG. 4

The spindle cells possess abundant glycogen particles (arrows) and rough endoplasmic reticula in their cytoplasm. ( $\times 7300$ )

emphasized by Jaffe and Lichtenstein (Jaffe et al., 1948) in their original description of the entity. Thorough curettage of the lesion is the treatment of choice, being curative in about 80 per cent of cases (Zillmer and Dorfman, 1989; Unni, 1996), although some patients do have local recurrence of the disease. While CMF may recur, malignant transformation of CMF has been documented rarely in the literature (Sehayik and Rosman, 1975). Complete en-bloc excision of the tumour, on the other hand, may give a lower incidence of recurrence (Schajowicz and Gallardo, 1971), but it is associated with considerably increased morbidity.

Chondromyxoid fibromas of the skull base, owing to its anatomical location, can pose diagnostic as well as therapeutic challenges to surgical pathologists, radiologists and surgeons. Apart from chondrosarcoma, chordoma is another differential diagnosis, which chrondromyxoid fibroma may closely resemble. CMF of the skull base, by virtue of its strategic location, is almost exclusively treated by curettage with, or without, post-operative radiotherapy. Very often it is not feasible to remove the tumour completely, therefore tumour recurrence is not unexpected.

The distinction between chondromyxoid fibroma, chondrosarcoma and chordoma has been thoroughly discussed in standard textbooks. When the tumour is adequately sampled. the pseudolobulated architecture with a peripheral hypercellularity and central fibromyxoid hypocellular area is highly characteristic of chondromyxoid fibroma. Well-differentiated chondrosarcoma can be distinguished from CMF by the better chondroid appearance of the tumour, together with the more overt cytological abnormality. Myxoid chondrosarcoma may be extremely difficult to distinguish from CMF if only a limited amount of material is available for examination. However, myxoid chondrosarcoma shows permeation into native bony trabeculae and it lacks the fibrous component of CMF. Chordoma can be distinguished from chondromyxoid fibroma by the presence of large epithelioid tumour cells with eosinophilic or vacuolated cytoplasm that are arranged in cords or cohesive nests. Immunohistochemical studies are extremely helpful in the distinction between chordoma and chondromyxoid fibroma. The former usually expresses EMA. S100 and cytokeratins, whereas the latter stains for $\$ 100$. but not cytokeratins nor EMA.

There have been only a few ultrastructural studies of chondromyxoid fibroma (Tornberg et al., 1973; Toremalm et al., 1976; Steiner et al.. 1979; Ushigome et al., 1982). The tumour cells have been found to show features of both chondroblastic and fibroblastic differentiation. Such dual differentiation is also observed histologically in the spindle fibroblastic cells in the present case, suggesting that the better differentiated chondroid cells and the spindle fibroblastic cells are of the same cell type. The positivity of both the chondroid cells and the fibroblastic cells for $\mathrm{S} 100$ protein is also in keeping with such an hypothesis. 


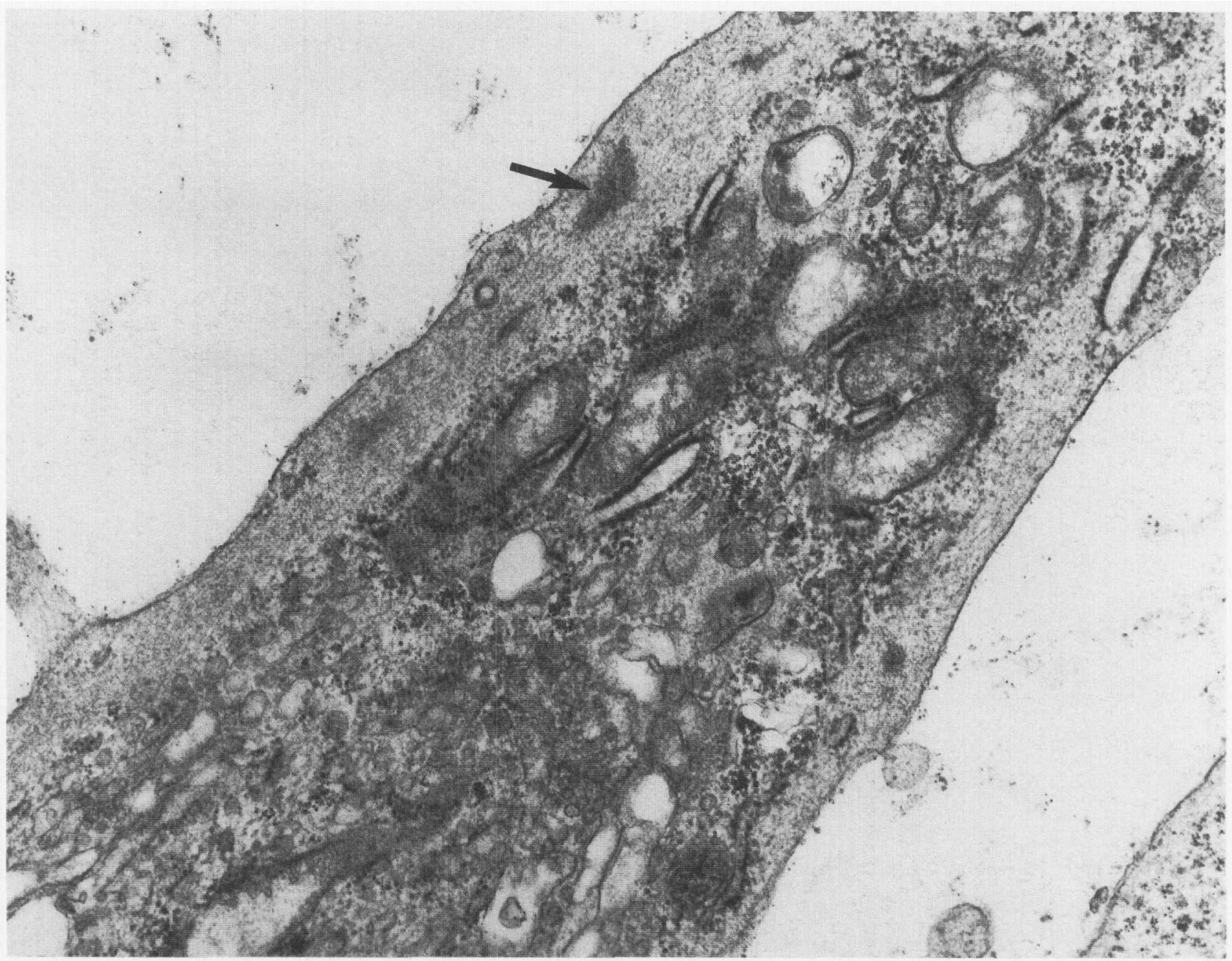

FIG. 5

Abundant rough endoplasmic reticula in the cytoplasm and small aggregates of microfilaments (arrow) beneath the cell membrane, suggestive of fibroblastic/myofibroblastic differentiation. $(\times 29000)$

In conclusion, we have documented an uncommon case of chondromyxoid fibroma of the skull base in a teenage girl. The tumour showed multiple recurrences over a period of 10 years after the initial operation. The histological appearances of the original and the three recurrent tumours are remarkably similar with no evidence of histological progression or dedifferentiation. Given the fact that it is practically impossible to temove the tumour completely, we believe this is the typical behaviour of chondromyxoid fibroma when it occurs at this site. The possible role of radiotherapy and chemotherapy as adjunctive therapies after surgery needs to be further explored and clarified.

\section{References}

Dahlin, D. (1956) Chondromyxoid fibroma of bone, with emphasis on its morphologic relationship to benign chondroblastoma. Cancer 9: 195-203.

Frank, E., Deruaz, J. P., de Tribolet, N. (1987) Chondromyxoid fibroma of the petrous-sphenoid junction. Surgical Neurology 27: 182-186.

Inoue, K., Sugiyama, Y., Ishii, R. (1978) Intrasellar fibromyxochondroma. Surgical Neurology 10: 167-170.

Jaffe, H., Lichtenstein, L. (1948) Chondromyxoid fibroma of bone: a distinction benign tumor likely to be mistaken especially for chondrosarcoma. Archives of Pathology 45: $541-551$.
Keel, S. B. Bhan, A. K., Liebsch, N. J., Rosenberg, A. E. (1997) Chondromyxoid fibroma of the skull base: a tumor which may be confused with chordoma and chondrosarcoma. American Journal of Surgical Pathology 21: 577-582.

Kitamura, R.. Gondo, M., Hiraharah. K., Hamada, H., Mihara, T.. Asakwa, T. (1982) A giant chondromyxoid fibroma originating from the right orbital roof. A case report. No Shinkei Geka 10: 731-736.

Maruyama, R., Nagaoka, S., Todaka, T., Nakahara, T., Kishida, K. (1994) Intracranial chondromyxoid fibroma extending into the jugular foramen. Pathology International 44: $857-859$.

Morikawa, E.. Sasaki, T., Basugi, N., Hashimoto, K., Iwata, J. (1987) Chondromyxoid fibroma of the skull-base extending from the middle fossa to the posterior fossa. Neurosurgery (Tokyo) 15: 1233-1238.

Patino-Cordoba, J. 1., Turner, J., McCarthy, S. W., Fagan, P. (1998) Chondromyxoid fibroma of the skull base. Otolaryngology - Head and Neck Surgery 18: 415-418.

Schajowicz, F., Gallardo, H. (1971) Chondromyxoid fibroma (fibromyxoid chondroma) of bone: a clinico-pathologic study of 32 cases. Journal of Bone and Joint Surgery [British] 53: 198-216.

Sehavik, S., Rosman, M. A. (1975) Malignant degeneration of a chondromyxoid fibroma in a child. Canadian Journal of Surgery 18: 354-360.

Steiner, G. C. (1979) Ultrastructure of benign cartilaginous tumors of intraosseous origin. Human Pathology 10: 1-86. 
Toremalm, N. G., Lindstrom C., Malm, L. (1976) Clinical records: Chondromyxoid fibroma of the pterygopalatine space. Journal of Laryngology and Otology 90: 971-978.

Tornberg, D. N., Rice, R. W., Johnston, A. D. (1973) The ultrastructure of chondromyxoid fibroma. Clinical Orthopaedics 95: 295-299.

Unni, K. K. (1996) Dahlin's Bone Tumor: General Aspects and Data on 11087 cases. 5th Edition. Lippincott-Raven, Philadelphia. pp 67-69.

Ushigome, S., Takakuwa, T., Shinagawa. T.. Kishida, H., Yamazaki, M. (1982) Chondromyxoid fibroma of bone: an electron microscopic observation. Acta Pathologica Japonica 32: 113-122.
Wu, C. T., Inwards. C. Y.. O'Laughlin, S. Rock, M. G. Beabout, J. W., Unni, K. K. (1998) Chondromyxoid fibroma of bone: a clinicopathologic review of 278 cases. Human Pathology 29: 438-446.

Zillmer, D., Dorfman. H. (1989) Chondromyxoid fibroma of bone: 36 cases with clinicopathologic correlation. Human Pathology 20: 952-964.

Dr Tony W. H. Shek.

Department of Pathology,

University of Hong Kong,

Queen Mary Hospital,

Hong Kong.

Fax: (852) 28728098 